\title{
ARCHAEOLOGICAL RESCUE EXCAVATION AND DIGITALIZATION OF CULTURAL HERITAGE
}

\author{
S. Varea ${ }^{\mathrm{a}}$, J.-B. Lemerle \\ ${ }^{a}$ Actual Foncier Topographie, Rouen, France - aft.varea@orange.fr \\ ${ }^{\mathrm{b}}$ AFT Archaeology, Rouen, France-jb.lemerle@aftopo.com
}

KEY WORDS: Archaeology, digitalization, cultural heritage, terrestrial laser scanning, conservation, 3D, Versailles, Pompei

\section{ABSTRACT:}

We present in this paper the original work and projects of AFT, a French company working in the complementary fields of topography, archaeological rescue excavation and digitalization of cultural heritage. Here are described more precisely the application of 3D scanning in archaeology, especially in rescue excavation, and the wish of the company to be ahead of its time in this field., followed by two examples, one in heritage object studies, the other in heritage building studies.

\section{1- RESCUE EXCAVATION}

AFT is at the origin an expert land surveyor and topographer, its office is situated in the urban area of Rouen (HauteNormandie). For about six years AFT has diversified its activities. Indeed, in addition of the classic activities of land surveyor and topographical surveys, AFT added in a first time photogrammetric and 3D digitalization activities which allowed it to work on patrimonial sites on a first time. On a second time, since summer 2012, AFT has created a new section of preventive archaeology approved by the French Ministry of Culture. There are now 3 departments: the main department for land survey and topography, another for $3 \mathrm{D}$ digitalization and heritage studies, and the last one for rescue excavations.

The department "AFT Archaeology" was approved by the French Ministry of Culture on the 22th of June 2012 after the achievement of a full technical report. This department counts 15 qualified archaeologists. This team is working throughout the French territory on Roman and Medieval archaeological sites.

Through the latest generation technological means, the will of the society is to be used in the field of Archaeology, challenging the traditional techniques used by professionals.

\section{2 - DIGITALIZATION TECHNIQUES}

3D scanning is a representation of space, according to our binocular vision and depth perception. The digital acquisition in three dimensions of an object allows, with current technologies, rendering scales, measures and identical meshes to the original object textures, following the three coordinates $\mathrm{X}, \mathrm{Y}$ and $\mathrm{Z}$. It consists of a recording spatial data acquired and restored digitally.

The lasergrammetry enable the position of points in a coordinate system. To enter and calculate these points into an X, $\mathrm{Y}$ and $\mathrm{Z}$ system, it is essential to obtain measures of distances and angular values.
The most common technique for portable scanners is the triangulation survey: following the distance between the scanner and a surface, the scanner uses a sensor to define the situation of a point. The laser spot sensor allows positioning with precision the coordinates of this point, and indeed creating the form of the digitized object.

Today's portable scanners offer a frequency up to 20000 measurements per second; these information are recorded by computer as coordinates of points in three dimensional space. The set of points products by 3D Scanners must thereafter be connected order to obtain a continuous surface, a "virtual object», containing or non colorimetric information.

AFT owns two scanners, representing two types of 3D laser scanning equipment: The LaserScan Focus 3D 120, by FARO, and the the handyscan ViewScan, by Creaform.

The LaserScan Focus 3D 120 by FARO, on tripod, is used for large surface laser surveys (statues, monuments, façades, archaeological sites, etc.).This appliance has a 120 meters scope; it allows to capture up 976000 points per second with a precision of 0,1 millimeter.

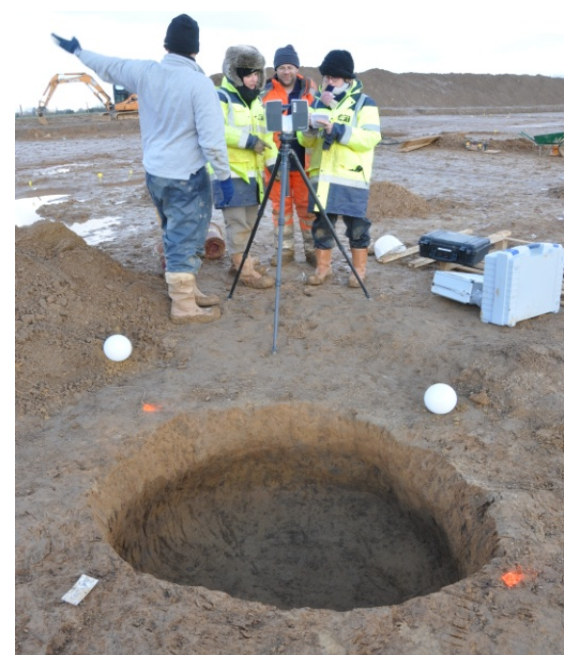

Figure 1. Archaeologists during digitalization 
Practically speaking, the device rotates on itself to $360^{\circ}$ horizontal; a mirror located on the device rotates $360^{\circ}$ vertically. Thus, the laser radiates its environment to $360^{\circ}$ in all directions (except a small shade under the tripod zone). The laser the scanner raises all obstacles he encounters around him. This allows to obtain a sphere point (generally 50 million points) in a radius of 120 meters around the appliance. It is possible to reduce the statement angle on a desired area (for example when working on façades or statues). Several stations are required in order to scan dead angles and zones of shadows, increasing the density of points and therefore the precision of statement. All stations will then assembled during posttreatment for obtaining a single points cloud. The points cloud will finally be transformed into mesh model.

The Handyscan ViewScan, a hand-held portable appliance, is used for movable objects, anthropological remnants and details. The VIUscan uses triangulation technique, and it operates on the principle of the geometry analysis of an object with laser and the self-positioning of the sensor. This self-positioning is made possible by the use of targets of stored records (which requires the provision of reflective patches on the object to be scanned). The uniqueness of this appliance is the simultaneous acquisition of the geometry, color and texture of the object. Indeed, in addition to the pair of laser defining normal at the intersection of their lines, the VIUscan has a third sensor for the acquisition of the color information immediately correctly positioned on the scanned object, preventing misplacement. An integrated scanner lighting system acquires colors as RGB standard. The handyscan VIUscan, class II according to French legislation (as well as a barcode reader), causes no danger to the scanned object (beyond a $700 \mathrm{~nm}$ wavelength, the radiation emitted by a laser can cause burns).

\section{APPLICATIONS}

\subsection{In rescue excavation context}

With this equipment, AFT can now offer a new approach to preventive archaeology. The laser scanning allows a built structures survey with a scientific precision, but the will of the company is to expand its use to hollow structures, very common in archaeology. Indeed, pits, postholes, or silos are part of daily archaeological work and need to be identified by drawings and topographic measurements

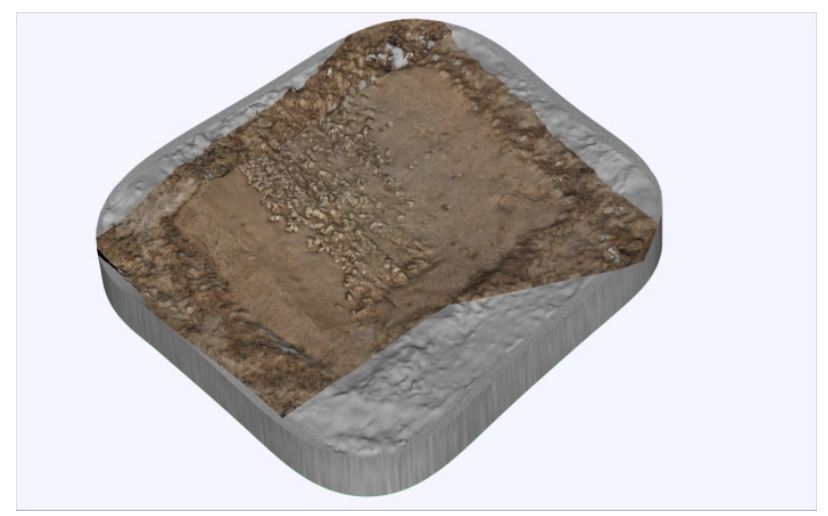

Figure 2. Digitalization of a Roman road in Flamanville.
These operations, carried out manually, can be time-consuming and depends on the subjectivity of human eye. The use of 3D scan would ease the task of the archaeologist while improving the measurements. In addition, the possibility of joining geographically and temporally scanned files, provides an overall picture of the site, following the archaeological research, and to better address the visual field and its structures.

Archaeology is a multidisciplinary science, and 3D can also be applied to many specialties. The archaeology of buildings, of course, is the first concerned by the use of 3D scanning, either for reading of ruins, masons' marks or viewing of old structures to reconstruct states of different constructions. Other areas, such as funerary archaeology, may be less likely to benefit from these techniques, but can benefit greatly. Scanning burials can put the individual in the context of specific discovery, without relying on a manual or photographic survey, which is twodimensional and is not ideal to fully investigate this type of structures.

Outside of work on the site, remember that archaeology is a scientific discipline where the majority of the study is done after the search by studying the documentation collected: surveys, drawings, photographs, objects and samples, and now scans. Here again the 3D scanner can address effectively and originally the work of the archaeologist. Digitalization is an efficient recording tool and shares great information. It provides measures and - a posteriori- information and access to the phases of a finished project, which is an important asset for the understanding of an excavation.

In addition, from a material point of view, scanning of archaeological material allows an exhaustive inventory of objects in the conservation status they have at the end of the operation. It also offers the opportunity to study objects remotely if needed, to extract measurements or details, preserving the fragile material, which should be handled as little as possible. One of the AFT Archaeology objectives is to create a database of archaeological artifacts in 3D, as a collection of virtual reference, whatsoever to the type of objects (ceramics, glass, metals...) or identification of specimens in archaeozoology as an example.

All this digital documentation can also serve the enhancement of the archaeological heritage and attract the attention of the public on the importance of rescue excavation. The great advantage of this technology is to provide access for the general public with visual medium easily understandable, and include it in a system which it is usually excluded.

\subsection{In heritage object studies: The petrified bodies of Pompeii}

Under the direction of the University of Valencia, an archaeologist-digitalizator of the AFT society went to Pompeii during August 2012. AFT worked for Llorenç ALAPONT, Archaeo-Antropologist, Director of the College of the bachelor and doctor in Archaeology of Valencia, and his team of students. Last year we digitalized 10 bodies with one children body wrapped in his sheet. The full bodies might be digitalized this year (2013) during a longer time. This kind of mission represents a long period of work. 
Llorenç ALAPONT and his team identified the petrified bodies. Those bodies were buried from a long time at the back of the Pompeian collections. Some of them were even lost.

More that the inventory, Llorenç ALAPONT carried out anthropological studies and pathological analysis of the bones which appeared from the bodies.

The goal of our $3 \mathrm{D}$ digitalization technique is to complete the studies of the anthropologist.

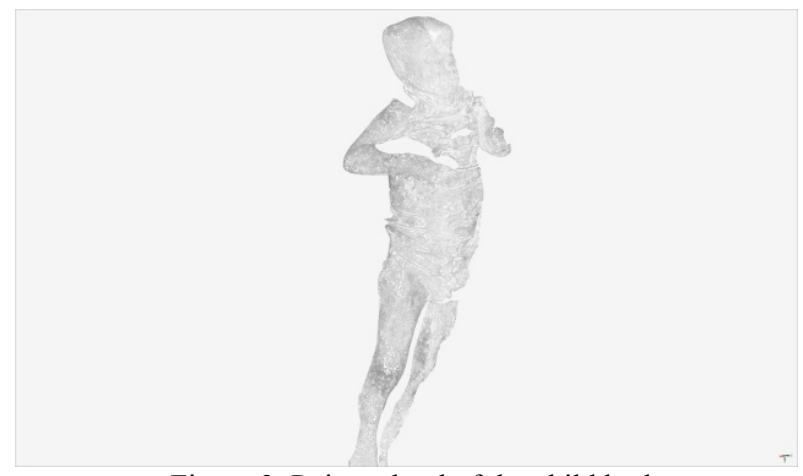

Figure 3. Points cloud of the child body

We digitalized the bodies one by one, placing them on a table around which we could turn. For each body, we made 10 positions ( 6 with the body on the back and the face looking at us, then 4 with the body on the face and the back looking at us). All these positions were necessary to obtain information and details we needed and also avoiding shaded areas (fig. 2).

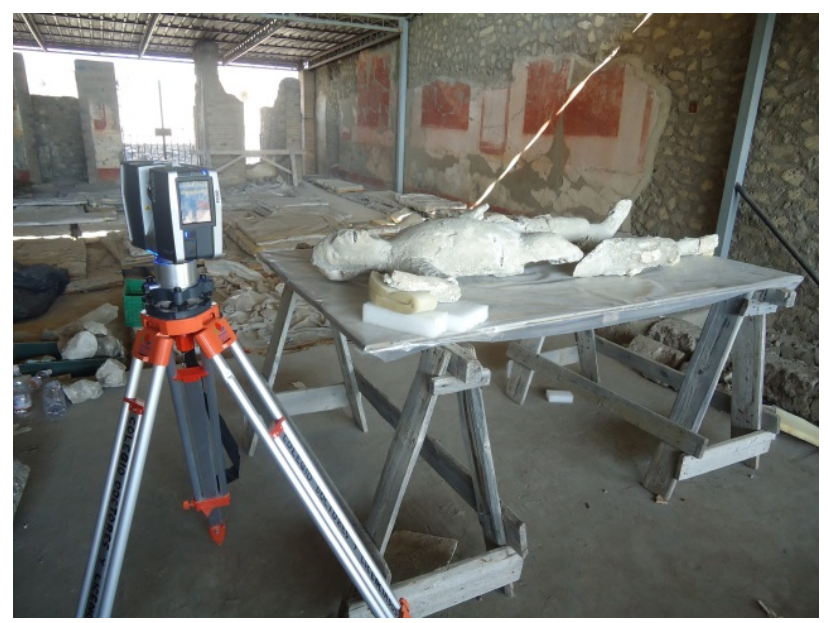

Figure 4. Digitalization of a Fugitive

The digitalization of museographical objects like petrified bodies have several applications and 3 main points of interests.

Firstly, the digitalization 3D allows the preservation of the bodies which is very important because they are falling into ruin. So our technique allows the record of all the bodies' datas in order to maintain them. Even if the bodies were destructed with the time and the problems of conservation, we will have a compliant digital copy (fig. 3).

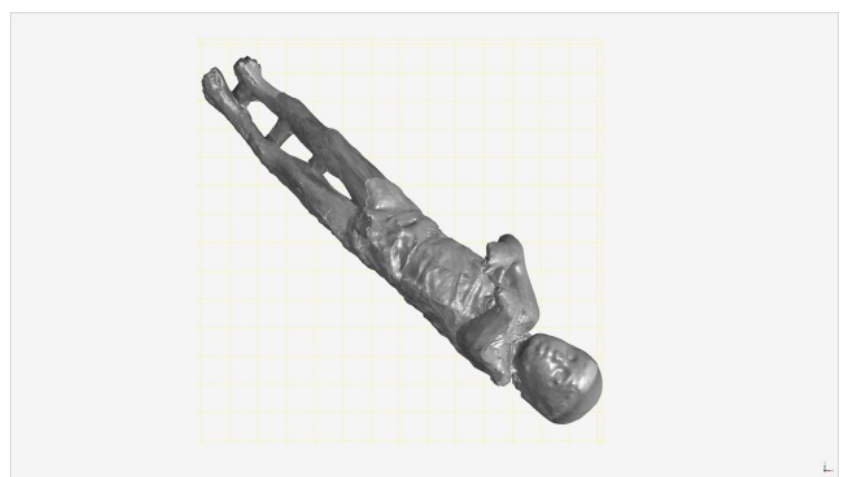

Figure 5. Final version of the digitalized body

Secondly, this technique gives a study support. The 3D models can be used by an anthropologist like Llorenç ALAPONT to get for example, the measurements and volume. We can also highlight the micro-details on the teeth or bones extremities to see precisely the pathology traces like degenerative osteoarthritis or dislocation bad-reduced.

Thirdly, we can offer our 3D models to present bodies to the public via internet or interactive hotspots. 3D modelization is a modern and novatrice way for mediation and patrimonial conservation.

After modelization of the Fugitives of Pompeii, we were able to measure skulls, teeth and bones with a great accuracy. Moreover, we can also present sections of bones. Concerning the research aspect of the modelization, it will probably allow a sexual discrimination between male and female, a restitution of Fugitives height, a study of past pathologies etc.

Otherwise, this mission was not conducted for research purpose only: the main aim was protecting patrimonial finds as bodies might deteriorate in near future. Modelization is one of the only way to preserve a maximum of information. Moreover, results will contribute to a restoration plan.

To sum up the interest of objects digitalization like bodies, we can conclude that it ensures the conservation of petrified bodies by creating a perfect, exact digital copy with highly accurate measurements. It also permits to realize very specific studies such as micro-topographical observations, section and intracranial volume calculations... Finally, the $3 \mathrm{D}$ model can be a base for broadcasting, valuation, museography or research problematic.

\subsection{In heritage building studies: The case of Versailles}

Under an order of the Public Institution "Versailles" and Google as part of its project Google Art Project, we conducted all surveys 3D with the Laser Scan FARO Focus 3D 120 on:

- The royal apartments and interior parts (king bedroom and apartments, the queen, the queen's office, the Royal Chapel, the "Galerie des Glaces", Opera, the coronation hall, lounge and Malachite Grand Trianon)

- All the statues of the castle walls

- The set of bronze statues and fountains of the gardens of Versailles. 


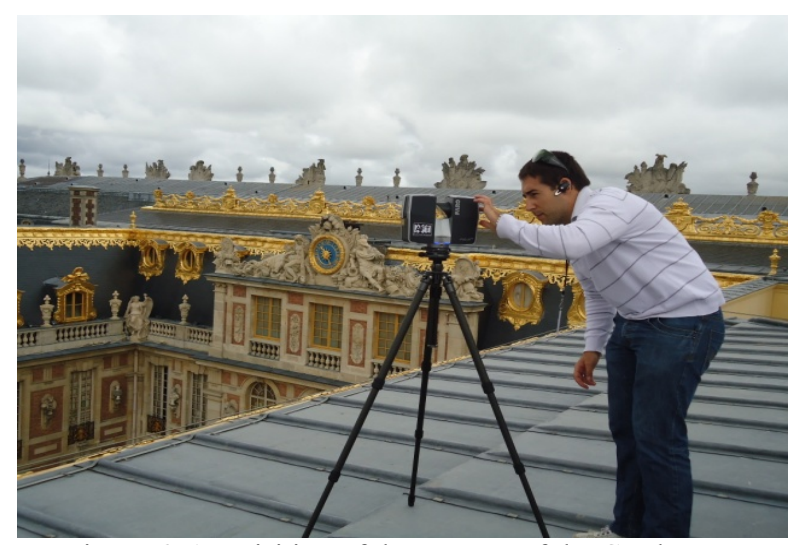

Figure 6. Acquisition of data on top of the Castle

The purpose of this project was to support virtual and interactive dissemination to the general public (via a comprehensive website) for a recovery operation of the castle and its dependencies (Figure 6).

AFT performed all scans and provide the raw data of the textured models who have been implemented in the interactive presentation of this. Due to the size of the site, the complexity of the context of digitization and rendering issues, two months on land were needed to enable us to carry out all the surveys, then 4 months of data processing have been necessary to produce roughly textured of the $3 \mathrm{D}$ models.

From June 2011 to August 2011, the scanning surveys were conducted with 120 3D Focus LaserScan of the brand FARO. A photographic coverage in high definition was made with a Nikon D90 camera. 3D survey was carried out in two stages: first the scanning stations, then a time data processing and assembly of the $3 \mathrm{D}$ model.

Sometimes complex situations (including the Hall of Mirrors) led us to question the relevance of path stations scans and methods and setting the device to adopt.

For apartments and interior parts, the objective was to identify comprehensively and accurately reliefs, colors and textures of the inner cladding: inlaid ceilings, moldings, wall tapestry

For the statues of marble and bronze fountains, the objective was to properly inform the volume of sculptures with the complexity of the land and subtlety of their materials. On top of that, these elements were not easily accessible. They were neither in the coronation of the attic cornice nor intersecting hedges or in the middle pool of the tire tread.

300 laser scanning stations were necessary for a complete record of Versailles and its dependencies. In general, the process of tracking stations used was defined by interior space (room of the queen, gallery...) then a central station was established, and a station at each end. This process is the same on a facade. The lightweight Laser scanner was easy to handle in the most inaccessible places (e.g. narrow rows in the opera, for statues on the outer ledges of the second floor).

For interior parts, four or five stations by scanning were made, part because of the shadows that the presence of furniture entails. The device was set to acquire directly after colorized laser scanning textures. Each piece has about 20 million colored points. Then the point cloud was converted to mesh model. The most telling example is the $3 \mathrm{D}$ modeling room of the queen.

For outside, most statues were relatively easy to scan as they are of modest size. Four stations at the four corners of each statue were taken each time. Depending on the size of the statue and special relief (arm position for example) shadows may result, so additional stations in the middle of all four sides were made. Some statues, especially along the great central aisle between the castle and the Grand Canal, are placed along fence, making it impossible to acquire data on the back of these statues. We had 3D models with a hole on the back. We had to deal with this problem by manually plugging the holes through our processing softwareure 2).

\section{Difficulties encountered:}

Sessions scanning requires a fairly strict and demanding environment. The laser scans any vegetation, fence, collapsed stone or any other items not belonging to the frame in place. By extension, it can hide and thus prevented the scan of some data. However, these error can sometimes be corrected by clouds of additional points. The additional elements are in turn removed during the phase of data processing.

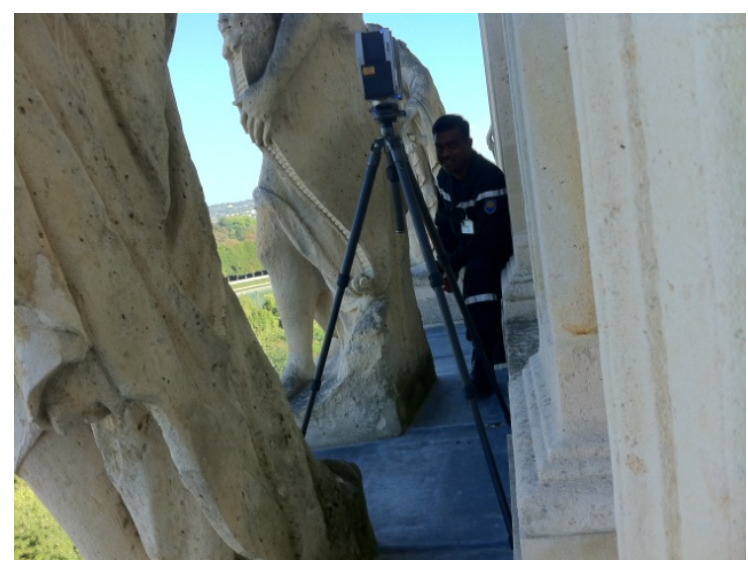

Figure 7. Technician in place

Public attendance was quite a significant barrier because when scanning should not be an absolute element starts between the camera and the subject scanner may distort decision point.

Other issues related to this intervention outside there was the distance data acquisition for some bronze fountains and statues on the ledges of the castle above the second floor. Indeed, bronze fountains were sometimes surrounded by large areas (including watersheds of Neptune and Apollo) preventing as close bronzes. The same is true for the statues of the pediments and cornices (triumphs for example) far from the scanner since the height of the walls. Indeed, larger distances could be misleading. All these settings caused the acquisition to take much longer periods in the field.

The latest issue of two months of full-time readings outside was weather since this type of device, because of the mirror and the laser cannot possibly work in the rain, even moderate. 


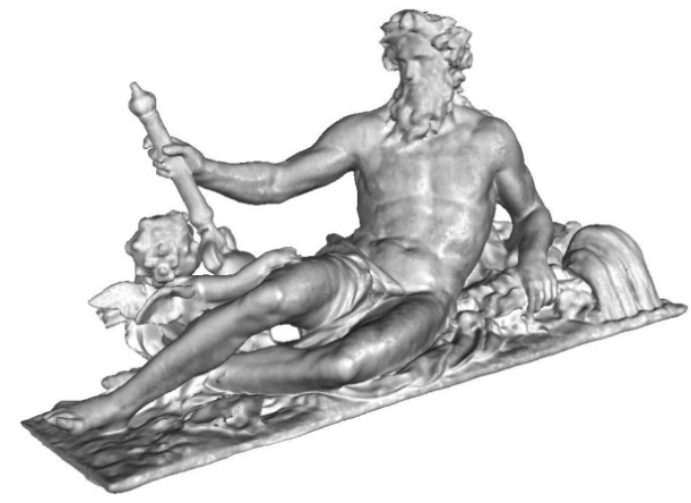

Figure 8. 3D model of a statue

Note that for the treatment of bronze statues and fountains, gardens, a technical constraint was imposed by Google. Indeed, our 3D models of statues should be legible on their Sketchup software to be integrated later on Streetview and Google Maps. The problem of opening the SketchUp 3D software models is that they must be converted into $3 \mathrm{ds}$ format. But this format only accepts models less than 65,000 polygons. This is binding as our 3D models of statues bronze fountains usually has 6 to 10 million polygons! So we were forced to optimize and degrade our templates to get to the bar 65000 triangles. Fortunately, this constraint was not imposed for interior parts. (Figure 9)

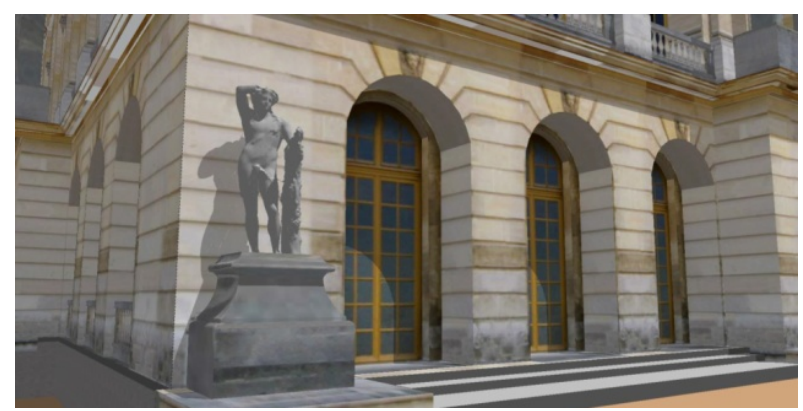

Figure 9. Publishing and development: provide the general public.
An accuracy of $0.005 \mathrm{~m}$ has been achieved for all the statues of Versailles (bronzes and marbles) and $0.001 \mathrm{~m}$ for all internal parts processed in true colors and textured. These models raw data were transmitted to computer graphics actors contracted by the public institution of the castle.

These 3D models were used by Versailles to offer interactive $3 \mathrm{D}$ presentations in twelve rooms "historic Versailles" at the entrance to the trail in the castle as well as their 3D Versailles internet site. As mentioned above, the 3D models of bronze statues and gardens are also directly visible on the Internet as well as Google Earth, Google Maps and Streetview. These models are shown on the website Versailles 3D.

\section{CONCLUSION}

AFT Archaeology is positioned as a new generation company in rescue excavations, willing to enroll in its time with the use of the technologies at its disposal. 3D scanning saves considerable time on the archaeological site and offers a large amount of accurate and easily manipulated data. The material available to professionals also fits the purpose, the dimensions of land or objects to be scanned, and finally gives an easily affordable representation of the evolution of the site.

However it should be noted that this technology is not without constraints. Besides fairly expensive 3D scanners -although their price tends to democratize- it takes on very powerful hardware, computers and software, to update regularly to follow the constantly changing world of digital. In addition, it is necessary to have in the field specialized stuff trained in the management of 3D points, or at least archaeologists with some training in 3D. For the treatment of data, however, one (or more) specialist in $3 \mathrm{D}$ data processing is essential.

AFT archeology is a young and dynamic company, and is currently implementing a methodology that includes all the parameters described above. For now, it's only in the early stages, but thanks to the skills of its employees and its innovative capabilities, AFT will be able to establish itself as one of the new standards of preventive archeology. 